\title{
MICROFABRICATED HIGH DENSITY MULTIPLEXED ELECTROSPRAY
}

\author{
C. M. Waits ${ }^{l}$, B. Morgan ${ }^{l}$, W. Deng ${ }^{2}$, N. R. Jankowski ${ }^{l}$, A. Gomez ${ }^{2}$, and B. Geil ${ }^{1}$ \\ ${ }^{1}$ US Army Research Laboratory, Adelphi, Maryland, USA \\ ${ }^{2}$ Yale University, New Haven, Connecticut, USA
}

\begin{abstract}
We present the fabrication, assembly, and testing of novel compact fuel atomization devices for portable power systems. Silicon multiplexed electrospray devices with nozzle densities $>11,500$ nozzles $/ \mathrm{cm}^{2}$ are demonstrated for the first time exhibiting liquid fluxes $>400 \mathrm{~mL} /$ hour $/ \mathrm{cm}^{2}$ with droplet diameters $<5 \mu \mathrm{m}$. Compared to prior electrospray devices, these results represent a $40 \mathrm{x}$ increase in nozzle density and a $50 \%$ reduction in driving voltage and droplet diameter enabling more efficient small-scale combustion.
\end{abstract}

\section{INTRODUCTION}

Small-scale portable power systems based on the combustion of liquid hydrocarbons have had increasing interest since the mid-1990's [1-3]. These combustion-based devices take advantage of the significantly higher energy density available in liquid hydrocarbons when compared to conventional batteries (at only $10 \%$ efficiency, diesel fuel can yield $4.2 \mathrm{MJ} / \mathrm{kg}, 10$ times more than the $0.36 \mathrm{MJ} / \mathrm{kg}$ for primary batteries). When scaling down the size of the combustor volume to the $\mathrm{cm}^{3}$ range and below, catalytic conversion and diffusion-controlled combustion will likely be used and require the fuel to be delivered as small and rapidly evaporating droplets [4]. Using liquid hydrocarbons such as JP-8, the logistical fuel of choice for the Army, a compact and reliable method to atomize the fuel has yet to be developed for portable power systems.

Multiplexed electrospray systems were previously demonstrated to be effective in atomizing liquid fuel into fine droplets enabling meso-scale combustion [4]. However, minimizing combustor volume requires smaller droplet diameters to reduce evaporation time and promote better mixing with the oxidizer. Smaller droplets can be obtained only at the cost of decreasing the flow rate per nozzle because of the monotonic dependence of droplet size on flow rate in typical electrospray operation. Therefore, maintaining or even increasing liquid flux levels requires increasing the level of multiplexing to counteract the smaller per nozzle flow rates.

Previous fabrication and assembly methods produced up to 250 nozzles $/ \mathrm{cm}^{2}$, but could not accommodate further scaling up of multiplexed electrospray devices for the small droplet diameters $(<10 \mu \mathrm{m})$ and higher flow rates $(>100 \mathrm{~mL} / \mathrm{hour})$ required in small-scale combustion applications [5-7]. Therefore, a new microfabrication and assembly technique was developed in this work to obtain a high density of electrospray sources that enables the smaller droplet diameters and larger flow rates. The operation of a 19-nozzle array is reported, demonstrating the capability of the high density multiplexed electrospray developed.

\section{MULTIPLEXED ELECTROSPRAY}

The design and configuration of a multiplexed electrospray was previously reported in [7]. The microfabricated multiplexed electrospray is composed of three layers: (1) a nozzle array, (2) an extractor electrode and (3) an electrically insulating spacer (Fig. 1). The device is operated with a potential drop between the conductive fluid within the nozzles and the extractor electrode (HV1-HV2). The fluid forms a cone at the nozzle tip through the balance of surface tension and the electrohydrodynamic force induced by the applied electric field. Above a critical field, a liquid ligament issues

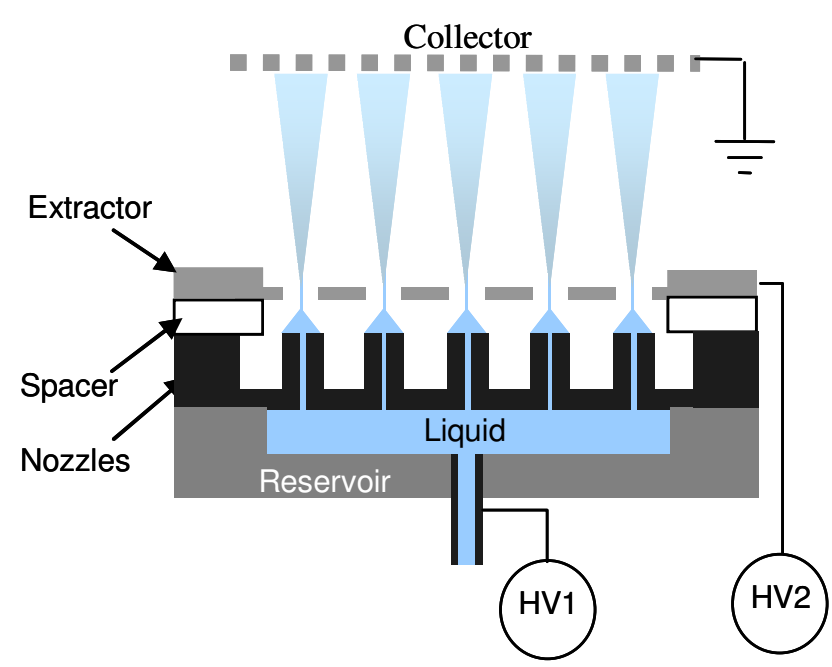

Figure 1: Schematic drawing of a multiplexed electrospray device and setup for testing. The grounded screen acts to draw the fuel droplets away from the device in place of a combustor or air flow.

from the cone tip and breaks up into monodispersed droplets. The Coulomb repulsion of the charged fluid causes the fluid droplets to disperse into a fine mist. A driving field was established between the extractor and grounded collector downstream (HV2-Gnd) to provide a removal force large enough to counteract the space charge effect that may cause satellite droplets to fly back and flood the electrode [8].

The critical geometric features of the multiplexed electrospray are shown in Table 1 for a nozzle array density greater than 11,500 nozzles $/ \mathrm{cm}^{2}$. Arrays of nozzles ranging from 1 to 91 nozzles were designed onto individual $1.6 \mathrm{~cm}^{2}$ die. The nozzle outer diameter, height, and pitch, combined with the spacer distance dictate the electric field at the tip of the liquid cone that enables the electrospray operation. Meanwhile, the nozzle inner diameter and length determine the fluidic characteristics of the device.

Table 1. High density multiplexed electrospray design quantities.

\begin{tabular}{|l|c|c|}
\hline Nozzle Parameters & Quantity & Units \\
\hline Outer diameter & 30 & $\mu \mathrm{m}$ \\
\hline Inner diameter & 15 & $\mu \mathrm{m}$ \\
\hline Height & 80 & $\mu \mathrm{m}$ \\
\hline Pitch & 100 & $\mu \mathrm{m}$ \\
\hline Extractor Parameters & & \\
\hline Hole diameter & 60 & $\mu \mathrm{m}$ \\
\hline Membrane thickness & 40 & $\mu \mathrm{m}$ \\
\hline Layer thickness & 200 & $\mu \mathrm{m}$ \\
\hline Spacer Parameters & & \\
\hline Spacer Distance & 60 & $\mu \mathrm{m}$ \\
\hline Anticipated electric field & $0.8-1.7$ & $\mathrm{x} 10^{5} \mathrm{~V} / \mathrm{cm}$ \\
\hline
\end{tabular}

Solid-State Sensors, Actuators, and Microsystems Workshop 
The spacer must provide electrical insulation with high electrical breakdown strength to support the electric field required to sustain the electrospray. Additionally, the spacer must provide mechanical stability between the extractor and nozzle layers and maintain micron-level alignment accuracy. Slight misalignment between the $30 \mu \mathrm{m}$ nozzles and the $60 \mu \mathrm{m}$ extractor holes may cause the dispersed sprays to impinge on the extractor and cause flooding. Quartz optical fibers were chosen as the spacing element with the dual purpose of providing good insulation as well as precise alignment capability when combined with alignment trenches.

\section{HIGH DENSITY ELECTROSPRAY FABRICATION}

The nozzle and extractor layers were fabricated on separate wafers and then bonded together at the die level using a novel optical fiber alignment technique and room temperature cured epoxy. Figure 2 illustrates the optimized fabrication process flow for the silicon nozzle and extractor layers.

The nozzle layer was fabricated using a 4-inch, $425 \mu \mathrm{m}$ $+/-25 \mu \mathrm{m}$, double sided polished silicon wafer with a $1.1 \mu \mathrm{m}$ thermally grown silicon dioxide layer. The first step in the nozzle fabrication defines the nozzle rings and alignment trenches into the silicon dioxide using photolithography and subsequent reactive ion etching (RIE). Then, a second photolithography step patterns only the inner hole of the nozzle into a thick photoresist masking layer. The inner holes were then etched using deep reactive ion etching (DRIE) to a depth greater of $100 \mu \mathrm{m}$. After this step, the backside silicon dioxide layer on the nozzle wafer was patterned with $60 \mu \mathrm{m}$ diameter holes. The larger diameter hole enables a higher etch rate, lower aspect ratio, and larger alignment tolerance than if a $15 \mu \mathrm{m}$ hole were used to match the inner diameter of the nozzle. The larger hole on the backside was etched to approximately $295 \mu \mathrm{m}$. The depths chosen for the nozzle inner diameter and backside hole create a $50 \mu \mathrm{m}$ thickness at the base of the nozzles after the $80 \mu \mathrm{m}$ nozzle etch. If the base becomes thinner than $50 \mu \mathrm{m}$ it may be too fragile for arrays having a large nozzle count.

The final fabrication step before dicing of the wafer was to etch the nozzles to the desired depth of $80 \mu \mathrm{m}$. Given the thin nozzle wall $(7.5 \mu \mathrm{m})$, and photolithography alignment tolerance $(1 \mu \mathrm{m})$, even a small negative taper creates a very thin sidewall at the bottom of the silicon nozzle. This can lead to punch through for sidewall angles as small as $\sim 86^{\circ}$ (Fig. 3a) or the nozzle breaking off during dicing and bonding. Tuning the deposition cycle time and the etch cycle time in the DRIE multiplexing process resulted in a nearly vertical sidewall profile, as shown in Fig. 3b. The DRIE process must be tuned for different pitches and nozzle outer/inner diameter combinations due to changing silicon loading conditions.

The extractor layer fabrication, shown in Fig. 2 was performed using a 4-inch, $200 \mu \mathrm{m}$, double sided polished silicon wafer with a $1.1 \mu \mathrm{m}$ silicon dioxide. The silicon dioxide layers were patterned using photolithography and RIE, while the silicon was etched using conventional DRIE. The extractor hole above the nozzle layer and the alignment trenches were etched together to a depth of $50 \mu \mathrm{m}$ to ensure the optical fiber does not touch the bottom of the trench during assembly, as well as ensure the topside etch breaks through. The topside was etched $155 \mu \mathrm{m}$, leaving a $45 \mu \mathrm{m}$ thick extractor membrane. The diameter of the extractor hole and the thickness of the membrane were designed leave excess space for the dispersed spray to pass through without impinging onto the sidewalls. The use of a $200 \mu \mathrm{m}$ wafer thickness allowed a smaller surface area on the sidewalls of the extractor, thereby minimizing possible space charge effects at the edge of the spray region.

\section{Nozzle Fabrication}

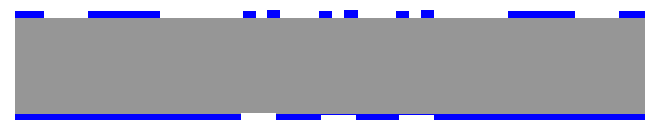

1. Pattern silicon dioxide for nozzles and alignment trenches

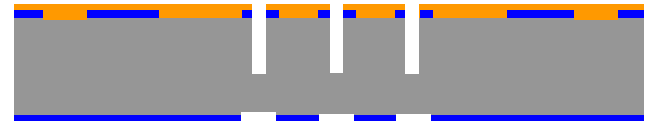

2. Pre-etch nozzle inner diameter using a patterned photoresist masking layer and DRIE.

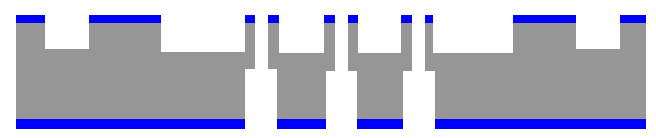

3. Nozzle formation using silicon dioxide masking layer and DRIE on both top and bottom. Alignment trench depths are defined at the same time.

\section{Extractor Fabrication}

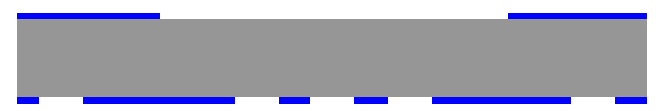

1. Pattern silicon dioxide on top to define cavity, and bottom to define extractor holes and alignment trenches.

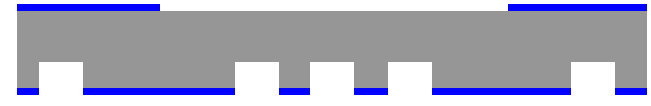

2. Etch extractor holes and alignment trenches using silicon dioxide masking layer and DRIE.

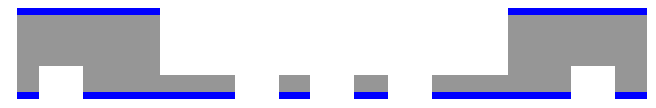

3. Define extractor membrane by etching cavity from the top using the silicon dioxide masking layer and DRIE.

\section{Silicon Silicon Dioxide Photoresist}

Figure 2. Fabrication flow for the nozzle and the extractor layers.

Conventional bond alignment techniques are limited to wafer level alignment and, furthermore, state-of-the-art machines can only achieve a $1 \mu \mathrm{m}$ alignment tolerance. In testing of the electrospray devices, it is convenient to employ an impermanent bond, which then requires bond alignment to be performed at the die level. A novel alignment and bonding technique was developed to assemble the nozzle array and extractor electrode layers with high alignment and gap precision. Our technique used $125 \mu \mathrm{m}$ diameter with $+/-1 \mu \mathrm{m}$ diameter tolerance bare optical fibers resting in opposing 


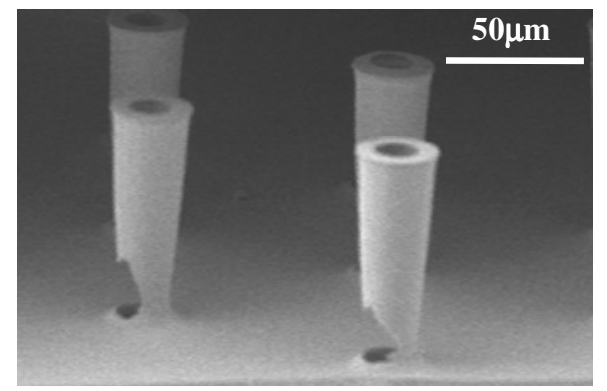

(a)

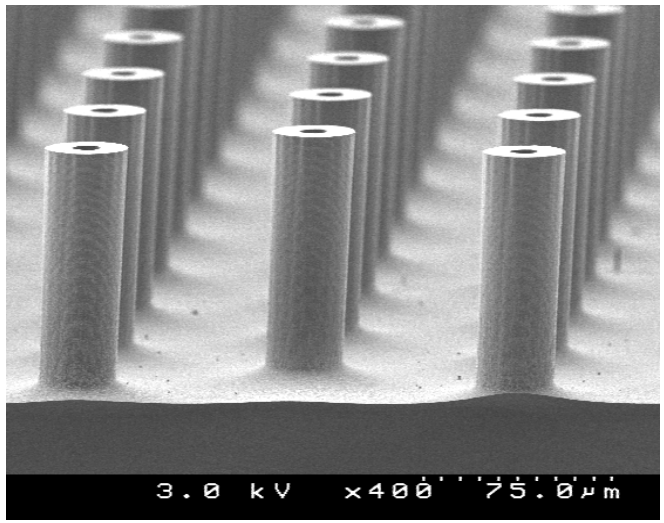

(b)

Figure 3. SEM images of fabricated $30 \mu \mathrm{m}$ O.D., $100 \mu \mathrm{m}$ pitch nozzle arrays for nozzles with (a) initial poor sidewall verticality and alignment and $(b)$ good sidewall verticality.

trenches, eliminating the need for specialized alignment equipment. The tight diameter tolerance of the optical fiber enables both alignment and gap control by lithographically tailoring the width of the alignment trenches, as shown in Fig. 4. The nozzle and extractor layers are then assembled using epoxy to secure the optical fibers in $108 \mu \mathrm{m}$ wide alignment trenches and achieve a gap of $60 \mu \mathrm{m}$ (Fig. 5).

\section{FABRICATION RESULTS AND TESTING}

Hexagonal arrays of 1, 7, 19, and 91 nozzles and matching extractors were fabricated having a pitch of $100 \mu \mathrm{m}$. Varying pitches can be accomplished using the same tuned DRIE etch recipe by including inactive nozzles to maintain silicon loading and aspect ratio. A cross-sectioned device having 91 nozzles is shown in Fig. 6 a with close-ups showing the optical fiber alignment and the nozzles in Fig. $6 \mathrm{~b}$ and $\mathrm{c}$ respectively.

The alignment accuracy and gap precision using the alignment trenches and the optical fiber were measured using an optical profilometer. The alignment accuracy was evaluated by how centered the nozzle was to the extractor opening when looking from the top of the extractor. The measurements showed in-plane alignment to be consistently below $1 \mu \mathrm{m}$ due to the self-centering nature of a cylindrical fiber within rectangular trenches. Gap uniformity across a $3.5 \mathrm{~mm}$ wide test membrane was measured to be $\Delta \mathrm{gap} \leq 1.5 \mu \mathrm{m}$. Potential errors in this alignment technique include the $+/-1 \mu \mathrm{m}$ optical fiber tolerance, fabrication accuracy of alignment trenches, and inconsistent contact between fiber and trench corners. Nonetheless, this new technique has proven quite simple and accurate as an insulating spacer and chip-level alignment/assembly technique.

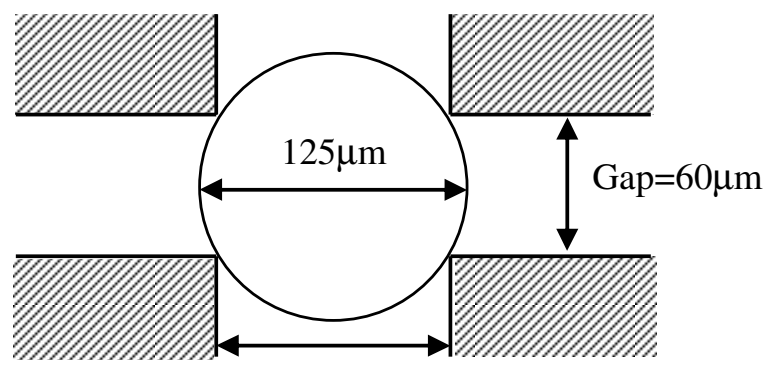

Trench width $=108 \mu \mathrm{m}$

Figure 4. Illustration of alignment and gap control using an optical fiber and tailored alignment trenches.

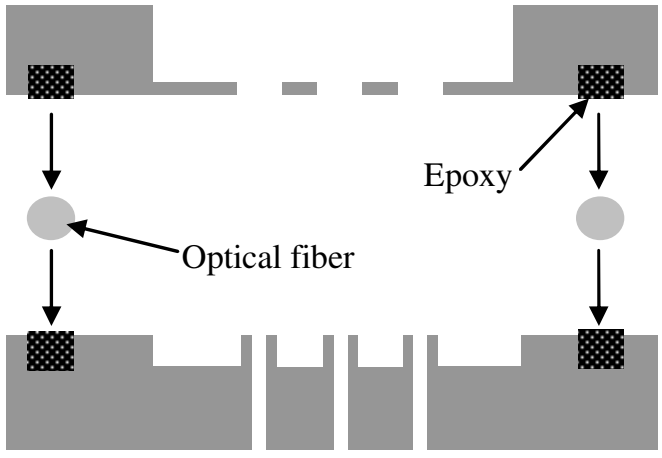

Figure 5. Illustration showing the assembly technique using an optical fiber to align and maintain a prescribed gap between nozzle and extractor layers.

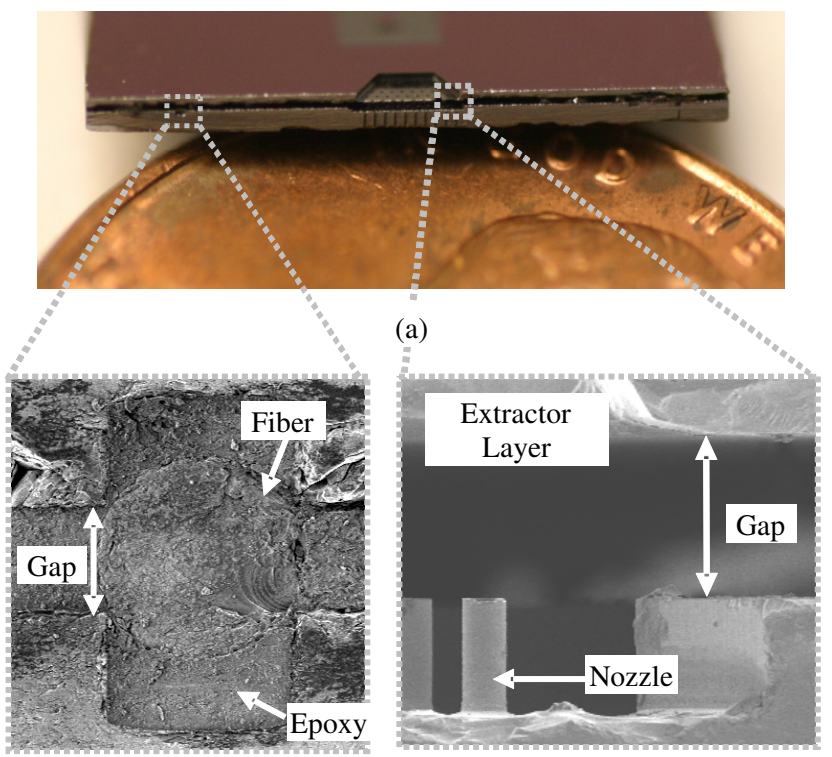

(b)

(c)

Figure 6. (a) Optical picture showing the cross-section of an assembled electrospray device and SEM images of $(b)$ the optical fiber alignment using the silicon trench and epoxy (see Figure 5) and $(c)$ gap between the nozzle and extractor layers. 
Successful electrospray operation with pure ethanol and flow rates on the order of $0.04 \mathrm{~mL} /$ hour per nozzle for a 19-nozzle, $100 \mu \mathrm{m}$ pitch array were achieved using a potential difference of $880 \mathrm{~V}$ between the nozzle layer and the extractor layer (HV1-HV2). The bias voltage between the grounded collector, shown in Fig. 1, and the nozzle layer was set to $5 \mathrm{kV}$ with the collector $5.5 \mathrm{~mm}$ away from the extractor layer, establishing a driving field of $9.1 \mathrm{kV} / \mathrm{cm}$. Figure 7 shows an optical image of the 19-nozzle device operating in electrospray mode.

An average droplet diameter of $4.5 \mu \mathrm{m}$ was measured using a TSI Phase Doppler Particle Analyzer (PDPA) downstream of the electrospray close to the collector using an equal-distance scan with a step of $100 \mu \mathrm{m}$. Figure 8 shows the average droplet diameter as the scan is stepped across the spray from A to A' in Fig. 7. The overall relative standard derivation of the diameter was $13 \%$, which is comparable to that of the uncertainty of the PDPA at $0.5 \mathrm{um}$, or $11 \%$. Furthermore, the spatial resolution of the optical technique may be inadequate to sort out primary droplets from satellites in each electrospray. As a result, the recorded spread in droplet size may be artificial and, in part, consequent to averaging both primary and satellite droplets in unequal proportion from point to point.

\section{CONCLUSION}

A new microfabrication process was developed for a high density of nozzles to be fabricated and assembled with an extractor electrode forming the multiplexed electrospray device. A novel alignment technique utilizing bare optical fibers was presented, with die level alignment accuracy below $1 \mu \mathrm{m}$ demonstrated. The new microfabrication process and assembly techniques presented enabled the first demonstration of a multiplexed electrospray having a source density greater than 11,500 nozzles $/ \mathrm{cm}^{2}$. Results of a 19-nozzle array showed a liquid flux greater than $400 \mathrm{~mL} / \mathrm{hour} / \mathrm{cm}^{2}$ with an average droplet diameter of $4.5 \mu \mathrm{m}$, which is sufficient for envisioned compact combustor volumes. Future work will focus on the integration of these electrospray fuel atomizers into Army portable power generator systems operating on JP-8.

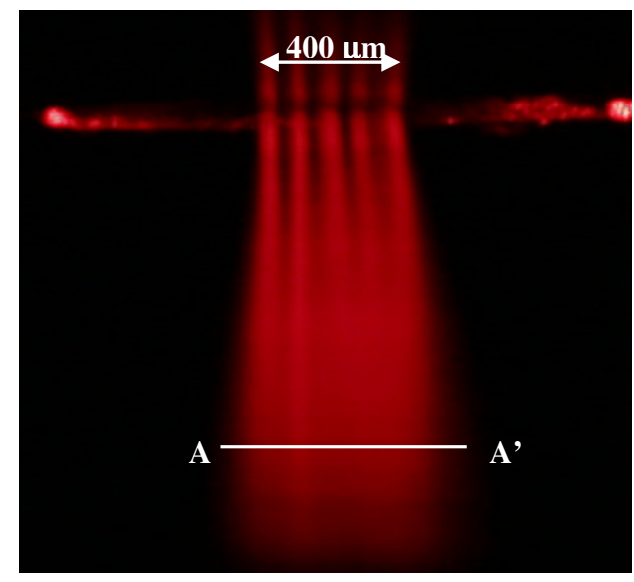

Figure 7. Optical photograph demonstrating electrospray action using pure ethanol and a 19-nozzle array at $>11,500$ nozzles $/ \mathrm{cm} 2$.

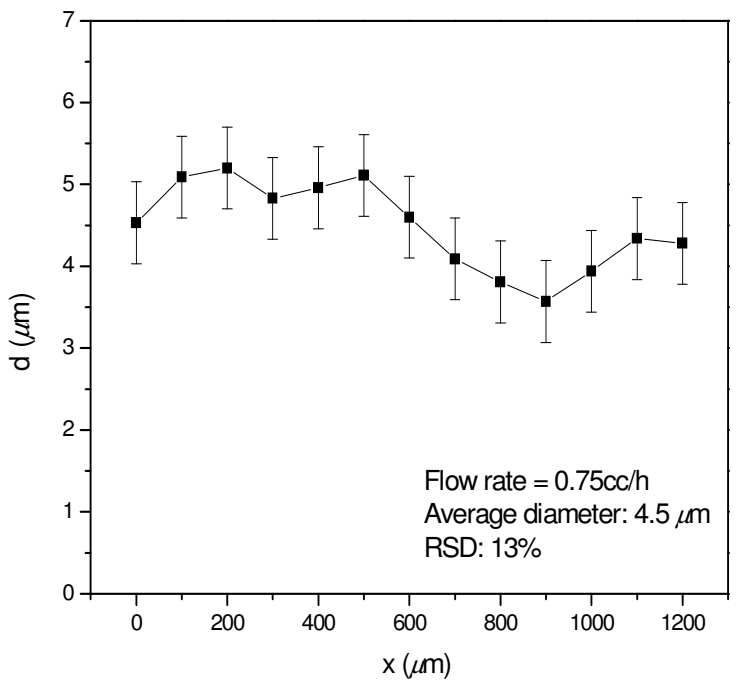

Figure 8. Scan from A-A' in Figure 7 showing the average droplet diameter is $4.5 \mu \mathrm{m}$ for a 19-nozzle, $100 \mu \mathrm{m}$ pitch nozzle array at $0.75 \mathrm{cc} / \mathrm{h}$.

\section{REFERENCES}

[1] A.H. Epstein, "Millimeter-scale, MEMS Gas Turbine Engines," Proceedings of ASME Turbo Expo 2003, Power for Land, Sea, and Air, June 16-19, Atlanta (2003), pp. 1-28.

[2] N. Müller and L.G. Fréchette, "Performance Analysis of Brayton and Rankine Cycle Microsystems for Portable Power Generation," Proceedings of IMECE2002, November 17-22, New Orleans (2002), pp. 1-10 .

[3] Y. Suzuki, Y. Okada, J. Ogawa, S. Sugiyama, and T. Toriyama, "Experimental study on mechanical power generation from MEMS internal combustion engine," Sensors \& Actuators: A. Physical, 141, 2, pp. 654-661 (2008).

[4] W. Deng, J.F. Klemic, X. Li, M. Reed, and A. Gomez, "Liquid Fuel Combustor Miniaturization via Microfabrication," Proceedings of the Combustion Institute, 31, pp. 2239-2246 (2007).

[5] Tang, K., Lin, T., Matson, D.W., Kim, T., and Smith, R.D., "Generation of multiple electrosprays using microfabricated emitter arrays for improved mass spectrometric sensitivity, Analytical Chemistry", Analytical Chemistry 73, pp. 1658-1663 (2001).

[6] R. Bocanegra, D. Galán, M. Márquez, I.G. Loscertales, and A. Barrero, "Multiple electrosprays emitted from an array of holes," Journal of Aerosol Science, 36, pp. 1387-1399 (2005).

[7] W. Deng, J.F. Klemic, X. Li, M.A. Reed, and A. Gomez, "Increase of electrospray throughput using multiplexed microfabricated sources for the scalable generation of monodisperse droplets," Journal of Aerosol Science, 37, pp. 696-714 (2006).

[8] W. Deng and A. Gomez, "Influence of space charge on the scale-up of multiplexed electrosprays," Journal of Aerosol Science, 38, pp. 1062-1078 (2007). 